\title{
Edaravone (Radicut), a free radical scavenger, is a potentially useful addition to thrombolytic therapy in patients with acute ischemic stroke (Review)
}

\author{
KIYOSHI KIKUCHI ${ }^{1-3}$, NAOKI MIURA ${ }^{4}$, KO-ICHI KAWAHARA ${ }^{5,6}$, YOSHINAKA MURAI $^{2}$, \\ MOTOHIRO MORIOKA ${ }^{3}$, PAULA. LAPCHAK ${ }^{1}$ and EIICHIRO TANAKA ${ }^{2}$
}

\begin{abstract}
${ }^{1}$ Department of Neurology, Cedars-Sinai Medical Center, Davis Research Building, Los Angeles, CA 90048, USA;
Departments of ${ }^{2}$ Physiology and ${ }^{3}$ Neurosurgery, Kurume University School of Medicine, Fukuoka 830-0011;

${ }^{3}$ Veterinary Teaching Hospital and Laboratory of Veterinary Diagnostic Imaging, Faculty of Agriculture, Kagoshima University, Kagoshima 890-0065; ${ }^{4}$ Laboratory of Functional Foods, Department of Biomedical Engineering, Osaka Institute of Technology, Osaka 535-8585; ${ }^{5}$ Department of Systems Biology in Thromboregulation, Kagoshima University Graduate School of Medical and Dental Sciences, Kagoshima 890-8520, Japan
\end{abstract}

Received July 3, 2012; Accepted August 8, 2012

DOI: $10.3892 / b r .2012 .7$

\begin{abstract}
Acute ischemic stroke (AIS) is a major cause of morbidity and mortality in the aging population worldwide. Alteplase, a recombinant tissue plasminogen activator, is the only Food and Drug Administration-approved thrombolytic agent for the treatment of AIS. Only 2-5\% of patients with stroke receive thrombolytic treatment, mainly due to delay in reaching the hospital. Edaravone is a free radical scavenger marketed in Japan to treat patients with AIS, who present within $24 \mathrm{~h}$ of the onset of symptoms. When used in combination with alteplase, edaravone may have three useful effects: enhancement of early recanalization, inhibition of
\end{abstract}

Correspondence to: Dr Eiichiro Tanaka, Department of Physiology, Kurume University School of Medicine, 67 Asahi-machi, Kurume, Fukuoka 830-0011, Japan

E-mail: eacht@med.kurume-u.ac.jp

Abbreviations: AIS, acute ischemic stroke; DEDAS, desmoteplase for acute ischemic stroke; DIAS, desmoteplase in acute ischemic stroke; ECASS III, European Cooperative Acute Stroke Study III; EDO, trial edaravone vs. sodium ozagrel in acute noncardioembolic ischemic stroke; EXTEND, extending the time for thrombolysis in emergency neurological deficits; HMGB1, high-mobility group box 1; J-ACT, Japan alteplase clinical trial; J-MARS, Japan post-marketing alteplase registration study; MMP, matrix metalloproteinase; PROTECT4.5, postmarketing registry on treatment with edaravone in acute cerebral infarction by the time window of $4.5 \mathrm{~h}$; SAINT, strokeacute ischemic NXY treatment; SITS-ISTR, safe implementation of thrombolysis in stroke international stroke thrombolysis registry; SITS-MOST, safe implementation of thrombolysis in strokemonitoring study; TTW, therapeutic time window; YAMATO, tissue type plasminogen activator and edaravone combination therapy

Key words: acute ischemic stroke, edaravone, alteplase, recombinant tissue plasminogen activator, thrombolytic agents alteplase-induced hemorrhagic transformation and extension of the therapeutic time window for alteplase. This is the first review of the literature evaluating the clinical efficacy of edaravone, aiming to clarify whether edaravone should be further evaluated for clinical use worldwide. This review covers both clinical and experimental studies conducted between 1994 and 2012. Edaravone is a potentially useful neurovascular protective agent, used in combination with thrombolytic agents to treat $>15$ million patients devastated by stroke worldwide annually. Additional clinical studies are necessary to verify the efficacy of edaravone when used in combination with a thrombolytic agent.

\section{Contents}

1. Introduction

2. Neuroprotective agents

3. Edaravone

4. Alteplase

5. Combination therapy using alteplase and edaravone

6. Newer thrombolytic agents

7. Conclusion

\section{Introduction}

Stroke, a major health problem, is the second most common cause of mortality worldwide (1). According to the World Health Organization, 15 million people experience stroke each year. Of these, 5 million succumb to the disease and another 5 million are permanently disabled (www.strokecenter.org/patients/stats.htm). Ischemic stroke is a major cause of adult mortality and disability, resulting in $>6$ million deaths annually (1).

Intravenous administration of the recombinant tissue plasminogen activator alteplase has been proven to improve 
the neurological outcome following an acute ischemic stroke (AIS). The only approved treatment for patients with AIS is the recanalization of the occluded cerebral arteries by thrombolysis using alteplase, within the first few hours subsequent to the onset of neurological symptoms (2-4). Although thrombolytic treatment with alteplase is beneficial, it must be delivered within a narrow 3-h therapeutic time window (TTW), or brain damage may be caused. Alteplase is neurotoxic, and its use carries a risk of hemorrhagic transformation or exacerbation of brain edema $(5,6)$. In practice, the narrow TTW is a major limiting factor in thrombolytic treatment using alteplase. At present, $<15-40 \%$ of patients with AIS arrive at the hospital early enough to receive this thrombolytic treatment (7-9), and only $2-5 \%$ of patients actually receive it $(10,11)$.

The internationally recommended dose of alteplase is $0.9 \mathrm{mg} / \mathrm{kg}$. However, only $0.6 \mathrm{mg} / \mathrm{kg}$ is currently used in Japan [http://www.jsts.gr.jp/guideline/048_051.pdf (in Japanese)], possibly causing reduced efficacy. Two large post-marketing surveys have been conducted: the safe implementation of thrombolysis in the stroke-monitoring study (SITS-MOST) and the Japan post-marketing alteplase registration study (J-MARS). In the SITS-MOST, the $0.9 \mathrm{mg} / \mathrm{kg}$ dose was evaluated in 6,483 European patients with AIS (12), while in the J-MARS the $0.6 \mathrm{mg} / \mathrm{kg}$ dose was evaluated in 7,492 Japanese patients with AIS (13). The rate of patients with complete recovery at 3 months was the same in the two studies (39\%). In J-MARS, the free radical scavenger edaravone was administered to $74.6 \%$ of the 7,492 patients (14). The combination with edaravone is therefore likely to have increased the effectiveness of the $0.6 \mathrm{mg} / \mathrm{kg}$ alteplase dose in Japanese patients with AIS in J-MRAS. In a recent study, Kimura et al (14) reported that edaravone enhanced recanalization in patients with AIS, who received thrombolytic treatment using alteplase.

This review aimed to present the currently available research findings regarding the use of edaravone, primarily in Japan, and to discuss potential further clinical studies aimed at assessing the usefulness of treatment with edaravone in combination with thrombolytic agents in patients with AIS worldwide.

\section{Neuroprotective agents}

Findings of a previous study estimated that more than 1,000 neuroprotective agents have been tested in animal models of ischemic stroke. However, none of the 114 neuroprotective agents that entered clinical trials were shown to be useful (15). Several free radical scavengers have been assessed for their efficacy in the treatment of AIS, yet few of these have shown success in trials conducted in Western countries. Several compounds have failed to prove clinical usefulness in the treatment of AIS $(15,16)$. Results of a large randomized trial, the stroke-acute ischemic NXY treatment I (SAINT I) trial (17), showed that NXY-059, a water-soluble free radical scavenger, reduced alteplase-induced cerebral hemorrhagic transformation. However, this scavenger is not approved for use in patients with AIS since no significant improvement was detected in the neurological outcomes in the SAINT II trial (18). By contrast, clinical trials of edaravone conducted in Japan showed better outcomes (16).

\section{Edaravone}

Edaravone $\left(\right.$ Radicut $^{\circledR}$ ) is a free radical scavenger marketed as a neurovascular protective agent in Japan by Mitsubishi Tanabe Pharma Corporation (Osaka, Japan) for the treatment of patients with AIS, who present within $24 \mathrm{~h}$ of the onset of symptoms (19). Edaravone was approved for the treatment of AIS in Japan in 2001, however, it remains under clinical investigation in various other countries (20). Edaravone is not currently approved for use in Western countries. However, edaravone is recommended by the American Heart Association in the guidelines for the early management of adults with AIS (21). Edaravone exerts antioxidant effects by inhibiting hydroxyl radical-dependent and -independent lipid peroxidation $(22,23)$. This antioxidant activity, the main suggested action mechanism, may protect against free radical-related injuries following AIS (24). Edaravone is a low-molecular-weight agent with water- and lipid-soluble properties, which readily crosses the blood-brain barrier, unlike other free radical scavengers $(25,26)$. These properties of edaravone may explain its neurovascular protective effects in patients with AIS, although other free radical scavengers have not shown such effects. Several lines of evidence have shown that edaravone has pleiotropic neurovascular protective effects subsequent to brain injury due to AIS (27-29). AIS is associated with the release of the high-mobility group box 1 (HMGB1), which causes apoptotic neuronal cell death (27). In a rat ischemic stroke model, edaravone was found to improve outcomes by attenuating the release of HMGB1 from the nuclei of neuronal cells (27). Kikuchi et al (28) hypothesized that edaravone may also reduce aquaporin-4 levels following an ischemic event, thereby reducing edema. Taken together, these findings indicate that edaravone may be useful for the treatment of patients with AIS, due to its being involved in inhibiting the deleterious molecular events associated with brain injury. Beyond AIS, edaravone has also been reported to have diverse protective effects against the ischemic injury of various other organs in several animal disease models (30-34).

The Japanese guidelines for the management of stroke 2009 suggest edaravone for the treatment of AIS as a grade B recommendation [http://www.jsts.gr.jp/guideline/057_059. pdf (in Japanese)]. Therefore, edaravone is now being widely used to treat AIS in Japan (14). Clinical trial data showed that administration of edaravone within $72 \mathrm{~h}$ of the onset of AIS significantly improves the neurological outcome over a 3-month follow-up period (35). In Japan, edaravone has been administered within $24 \mathrm{~h}$ of the onset of AIS in patients with multiple stroke subtypes, such as lacunae, large-artery atherosclerosis and cardioembolic stroke (36-38).

For patients with non-cardioembolic AIS, the Japanese guidelines for the management of stroke 2009 suggested the use of the antiplatelet agent sodium ozagrel (ozagrel), as a grade B recommendation [http://www.jsts.gr.jp/guideline/055_056.pdf (in Japanese)]. Ozagrel and edaravone are used in combination in the treatment of several patients with non-cardioembolic AIS in Japan. In their study, Shinohara et al (37) directly compared the effects of these two medications in 401 patients with AIS in the edaravone vs. sodium ozagrel in acute noncardioembolic ischemic stroke (EDO trial). The 'no symptoms' 
and 'no significant disability' patient rate in the edavarone and ozagrel groups at 3 months was 57.1 and $50.3 \%$, respectively. Thus, edaravone was at least as effective as ozagrel for the treatment of non-cardioembolic AIS.

The results of two clinical trials suggested a correlation between the duration of edaravone therapy and its efficacy. The duration of edaravone therapy affected the functional outcome and muscle atrophy in patients with AIS. While Naritomi et al (36) suggested that edaravone be administered for a longer time period to improve functional outcome, Unno et al (38) suggested the same to decrease muscle atrophy due to disuse.

\section{Alteplase}

The findings of recent studies suggested that the TTW for intravenous alteplase to treat AIS may be extended from 3 to $4.5 \mathrm{~h}$. The safe implementation of thrombolysis in stroke international stroke thrombolysis registry (SITS-ISTR) (39) and the European Cooperative Acute Stroke Study III (ECASS III) (40) showed that thrombolysis is beneficial if administered within the first $4.5 \mathrm{~h}$ after the onset of the symptoms. A recent meta-analysis of 12 randomized trials found that the administration of intravenous alteplase within $6 \mathrm{~h}$ after the onset of AIS improved functional outcome (41). The extending of the time for thrombolysis in emergency neurological deficit (EXTEND) clinical trial is ongoing. This trial aims to analyze 400 patients who received intravenous alteplase within $9 \mathrm{~h}$ after the onset of stroke symptoms (http://clinicaltrials.gov/ct2/show/NCT00887328). The TTW for intravenous alteplase may therefore be extended to $4.5 \mathrm{~h}$ or even longer in the future. However, further extension beyond this time period is unlikely, due to an increased risk of serious side effects. Delayed thrombolysis may be associated with an increased risk of hemorrhagic transformation, brain edema and additional side effects (42). Any treatment that reduces the risk of alteplase-associated neurovascular injury may therefore extend the TTW for a safe and effective reperfusion therapy, while ultimately increasing the overall efficacy of thrombolytic therapy using alteplase. The use of a neurovascular protective drug in combination with alteplase may increase in the future.

\section{Combination therapy using alteplase and edaravone}

Kimura et al (14) have recently reported that administration of edaravone during alteplase infusion is likely to enhance recanalization in patients with AIS. Of the 40 patients enrolled in their study, 23 were assigned to the edaravone group (intravenous alteplase infusion with simultaneous intravenous edaravone) and 17 to the non-edaravone group (intravenous alteplase infusion followed by intravenous edaravone). Early recanalization occurred more frequently in the edaravone group $(56.5 \%)$ compared to the non-edaravone group (11.8\%). The rate of patients with 'remarkable' or 'good' recovery was significantly higher in the edaravone $(80.1 \%)$ compared to the non-edaravone group (45.5\%). Kimura et al (14) hypothesized that the administration of edaravone during alteplase infusion inhibited endothelial cell injury in the occluded artery, and maintained the release of the tissue plasminogen activator from endothelial cells, thereby enhancing early recanalization.
In the ischemic brain, reperfusion using alteplase occasionally causes catastrophic hemorrhagic transformation (43). Certain research groups have reported that the administration of edaravone increased the survival rate, and inhibited alteplase-induced hemorrhagic transformation, blood-brain barrier damage and oxidative stress in the ischemic brains of rats, and hypothesized that edaravone may reduce the activity of matrix metalloproteinase-9 (MMP-9) (43-46). In their study, Kano et al (47) reported that edaravone attenuated the extravasation of alteplase through the cerebral vessels in a rat model of AIS. Combination treatment with edaravone is therefore expected to extend the TTW for the use of alteplase (46). Furthermore, Isahaya et al (48) reported that edaravone treatment was associated with lower MMP-9 serum levels in patients with AIS.

In a rat model of acute intracerebral hemorrhage, the administration of edaravone attenuated neurological deficits, brain edema, oxidative injury and iron- and thrombin-induced neuronal injury (49). Edaravone, therefore, inhibited hemorrhagic transformation, while attenuating brain injury subsequent to hemorrhage.

The rate of patients with symptomatic intracerebral hemorrhage subsequent to alteplase infusion was negatively correlated with the rate of combined treatment with edaravone in several clinical trials. The rate of combined treatment with edaravone was $0 \%(0 / 6,483$ patients) in SITS-MOST $(12,50,51), 0 \%(0 / 103$ patients) in the Japan alteplase clinical trial (J-ACT) $(50,52), 74.2 \%(5,557 / 7,492$ patients) in J-MARS $(13,50)$ and $91.4 \%(53 / 58$ patients) in the J-ACT II trial $(50,53)$. The rate of symptomatic intracerebral hemorrhage was $8.5 \%$ in SITS-MOST (12,51), 5.8\% in J-ACT (52), 3.5\% in J-MARS (13) and $0 \%$ in J-ACT II (53). These results indicate that edaravone treatment may inhibit hemorrhagic transformation in patients with AIS who receive thrombolytic treatment using alteplase.

Edaravone has beneficial effects when used in combination with alteplase. Furthermore, the timing of edaravone administration (prior to, during or subsequent to alteplase infusion) is extremely important. In Japan, two ongoing clinical trials are likely to reveal the most effective timing for edaravone administration by investigating 10,000 patients with AIS, undergoing thrombolytic treatment using alteplase: the postmarketing registry on treatment with edaravone in acute cerebral infarction by the time window of $4.5 \mathrm{~h}$ (PROTECT4.5) trial [http://medical.mt-pharma.co.jp/di/radicut/rct-protect.pdf (in Japanese)] and the tissue type plasminogen activator and edaravone combination therapy (YAMATO) study [http:// www.kawasaki-m.ac.jp/yamato/ (in Japanese)].

\section{Newer thrombolytic agents}

In a significant number of patients, intravenous thrombolysis using the second generation thrombolytic agent alteplase is ineffective. Patients with proximal occlusion or a large thrombus have lower rates of recanalization (54). Alteplase has several shortcomings: it increases the risk of hemorrhage, it is suppressed by the plasminogen-activator inhibitor and it has neurotoxic side effects (54). Newer thrombolytic agents, such as tenecteplase, reteplase and desmoteplase, offer pharmacokinetic and hemodynamic advantages over alteplase and have 
been, or are currently being, tested for safety and efficacy in clinical trials $(54,55)$.

Tenecteplase, a third generation thrombolytic agent, is a genetically-engineered mutant tissue plasminogen activator, with pharmacokinetic advantages over alteplase (56). Tenecteplase offers high fibrin selectivity, a long half-life, as well as an increased thrombolytic potency on platelet-rich clots (57). It conserves fibrinogen at therapeutic doses and is not inhibited by the plasminogen-activator inhibitor (57). Parsons et al (58) recently reported a phase IIB trial, which found that tenecteplase was associated with a markedly better reperfusion and clinical outcomes, without a significant difference in cerebral hemorrhage when compared to alteplase, in 75 patients treated within $6 \mathrm{~h}$ after the onset of AIS.

Reteplase, a third generation thrombolytic agent, is a recombinant non-glycosylated form of alteplase, with a longer half-life and better thrombus penetration compared to alteplase (57). However, reteplase seems to cause a greater adenosine diphosphate- and thrombin-induced platelet aggregation and a greater glycoprotein IIb/IIIa expression compared to alteplase, which is theoretically a disadvantage (54). Recently, Misra et al (59) reported that high intra-arterial doses of reteplase proved to be safe when administered with or without mechanical thrombolysis in 197 patients with AIS, also undergoing a full-dose intravenous alteplase treatment.

Desmoteplase (Desmodus rotundus salivary plasminogen activator) is a fourth generation thrombolytic agent with several theoretical advantages. It demonstrates minimal neurotoxicity, a high selectivity and specificity for fibrin and a long half-life, allowing for it to be administered as a single bolus $(60,61)$. Two phase II trials demonstrated its safety and efficacy: desmoteplase in acute ischemic stroke (DIAS) (62) and desmoteplase for acute ischemic stroke (DEDAS) (63). However, the DIAS-2 phase III trial did not replicate the positive efficacy findings of the phase II trial (64). Additional randomized phase III clinical trials (DIAS-3 and DIAS-4) are currently being conducted, with a planned sample size of 320 patients with vessel occlusion or high-grade stenosis of the proximal cerebral arteries on MRI or CT-angiography (65).

At present, the evidence for clinically effective thrombolysis in the treatment of AIS is the strongest for tenecteplase, although more effective agents are currently being investigated. As edaravone may have beneficial effects when used in combination with newer thrombolytic agents, it should be further investigated in future clinical trials. Currently, the combination of edaravone and alteplase is likely to be the optimal treatment for patients with AIS, although the combination of edaravone with one of the newer thrombolytic agents may be even more effective.

\section{Conclusion}

To increase the number of patients with AIS, able to benefit from the alteplase treatment in the future, the inhibition of hemorrhagic transformation and the extension of the TTW are extremely important. At present, the only neurovascular protective agent to achieve these effects is edaravone. Furthermore, edaravone enhances the effectiveness of recanalization by alteplase. However, the currently available global clinical evidence for the efficacy of edaravone is inadequate.
A large-scale multi-arm clinical trial to evaluate combination therapy in Western countries should be conducted if the findings of the previous study showing the efficacy of edaravone can be reproduced. The potential clinical usefulness of edaravone in a neurovascular protective treatment should be examined in combination with thrombolytic treatment using various agents, such as alteplase, tenecteplase, reteplase and desmoteplase. Edaravone needs to be further studied in a well-controlled clinical trial using a standard dose, treatment duration and time to unequivocally ascertain its efficacy. In a clinical trial of the combination therapy using edaravone and various thrombolytic agents, the initial goal should be to provide evidence of the inhibition of hemorrhagic transformation, the extension of the TTW for the thrombolytic agent and enhanced recanalization.

\section{Acknowledgements}

This review was supported by grants from SENSHIN Medical Research Foundation. The authors would like to thank Drs Yoko Tsurusaki, Sachiko Nakashima, Rumi Ito and Tomoko Matsuo of the Kurume University for their valuable assistance.

\section{References}

1. Ingall T: Stroke - incidence, mortality, morbidity and risk. J Insur Med 36: 143-152, 2004.

2. Tissue plasminogen activator for acute ischemic stroke. The National Institute of Neurological Disorders and Stroke rt-PA Stroke Study Group. N Eng J Med 333: 1581-1587, 1995.

3. European Stroke Organisation Exective Committee: Guidelines for management of ischaemic stroke and transient ischaemic attack 2008. Cerebrovasc Dis 25: 457-507, 2008.

4. Adams HP Jr, del Zoppo G, Alberts MJ, et al: Guidelines for the early management of adults with ischemic stroke: a guideline from the American Heart Association/American Stroke Association Stroke Council, Clinical Cardiology Council, Cardiovascular Radiology and Intervention Council, and the Atherosclerotic Peripheral Vascular Disease and Quality of Care Outcomes in Research Interdisciplinary Working Groups: The American Academy of Neurology affirms the value of this guideline as an educational tool for neurologists. Circulation 115: e478-e534, 2007.

5. Cheng T, Petraglia AL, Li Z, et al: Activated protein C inhibits tissue plasminogen activator-induced brain hemorrhage. Nat Med 12: 1278-1285, 2006.

6. Nicole O, Docagne F, Ali C, et al: The proteolytic activity of tissue-plasminogen activator enhances NMDA receptor-mediated signaling. Nat Med 7: 59-64, 2001.

7. Katzan IL, Hammer MD, Hixson ED, Furlan AJ, Abou-Chebl A and Nadzam DM: Utilization of intravenous tissue plasminogen activator for acute ischemic stroke. Arch Neurol 61: 346-350, 2004.

8. Lichtman JH, Watanabe E, Allen NB, Jones SB, Dostal J and Goldstein LB: Hospital arrival time and intravenous t-PA use in US Academic Medical Centers, 2001-2004. Stroke 40: 3845 $3850,2009$.

9. Reeves MJ, Broderick JP, Frankel M, et al: The Paul Coverdell National Acute Stroke Registry: initial results from four prototypes. Am J Prev Med 31: S202-S209, 2006.

10. California Acute Stroke Pilot Registry (CASPR) Investigators: Prioritizing interventions to improve rates of thrombolysis for ischemic stroke. Neurology 64: 654-659, 2005.

11. Albers GW and Olivot JM: Intravenous alteplase for ischaemic stroke. Lancet 369: 249-250, 2007.

12. Wahlgren N, Ahmed N, Davalos A, et al: Thrombolysis with alteplase for acute ischaemic stroke in the Safe Implementation of Thrombolysis in Stroke-Monitoring Study (SITS-MOST): an observational study. Lancet 369: 275-282, 2007.

13. Nakagawara J, Minematsu K, Okada Y, et al: Thrombolysis with $0.6 \mathrm{mg} / \mathrm{kg}$ intravenous alteplase for acute ischemic stroke in routine clinical practice: the Japan post-Marketing Alteplase Registration Study (J-MARS). Stroke 41: 1984-1989, 2010. 
14. Kimura K, Aoki J, Sakamoto Y, et al: Administration of edaravone, a free radical scavenger, during t-PA infusion can enhance early recanalization in acute stroke patients - a preliminary study. J Neurol Sci 313: 132-136, 2012.

15. No authors listed: Neuroprotection: the end of an era? Lancet 368: 1548, 2006

16. Lapchak PA and Zivin JA: The lipophilic multifunctional antioxidant edaravone (radicut) improves behavior following embolic strokes in rabbits: a combination therapy study with tissue plasminogen activator. Exp Neurol 215: 95-100, 2009.

17. Lees KR, Zivin JA, Ashwood T, et al: NXY-059 for acute ischemic stroke. N Eng J Med 354: 588-600, 2006.

18. Shuaib A, Lees KR, Lyden P, et al: NXY-059 for the treatment of acute ischemic stroke. N Eng J Med 357: 562-571, 2007.

19. Lapchak PA: A critical assessment of edaravone acute ischemic stroke efficacy trials: is edaravone an effective neuroprotective therapy? Expert Opin Pharmacother 11: 1753-1763, 2010.

20. Wang CX and Shuaib A: Neuroprotective effects of free radical scavengers in stroke. Drugs Aging 24: 537-546, 2007.

21. Adams HP Jr, del Zoppo G, Alberts MJ, et al: Guidelines for the early management of adults with ischemic stroke: a guideline from the American Heart Association/American Stroke Association Stroke Council, Clinical Cardiology Council, Cardiovascular Radiology and Intervention Council, and the Atherosclerotic Peripheral Vascular Disease and Quality of Care Outcomes in Research Interdisciplinary Working Groups: the American Academy of Neurology affirms the value of this guideline as an educational tool for neurologists. Stroke 38: 1655-1711, 2007.

22. Watanabe T, Yuki S, Egawa M and Nishi H: Protective effects of MCI-186 on cerebral ischemia: possible involvement of free radical scavenging and antioxidant actions. J Pharmacol Exp Ther 268: 1597-1604, 1994.

23. Yamamoto T, Yuki S, Watanabe T, Mitsuka M, Saito KI and Kogure K: Delayed neuronal death prevented by inhibition of increased hydroxyl radical formation in a transient cerebral ischemia. Brain Res 762: 240-242, 1997.

24. Lapchak PA and Araujo DM: Development of the nitrone-based spin trap agent NXY-059 to treat acute ischemic stroke. CNS Drug Rev 9: 253-262, 2003.

25. Watanabe $\mathrm{T}$, Tahara $\mathrm{M}$ and Todo $\mathrm{S}$ : The novel antioxidant edaravone: from bench to bedside. Cardiovasc Ther 26: 101-114, 2008

26. Yoshida H, Yanai H, Namiki Y, Fukatsu-Sasaki K, Furutani N and Tada N: Neuroprotective effects of edaravone: a novel free radical scavenger in cerebrovascular injury. CNS Drug Rev 12 9-20, 2006

27. Kikuchi K, Kawahara K, Tancharoen S, et al: The free radical scavenger edaravone rescues rats from cerebral infarction by attenuating the release of high-mobility group box-1 in neuronal cells. J Pharmacol Exp Ther 329: 865-874, 2009.

28. Kikuchi K, Tancharoen S, Matsuda F, et al: Edaravone attenuates cerebral ischemic injury by suppressing aquaporin-4. Biochem Biophys Res Commun 390: 1121-1125, 2009.

29. Tanaka E, Niiyama S, Sato S, Yamada A and Higashi $\mathrm{H}$ Arachidonic acid metabolites contribute to the irreversible depolarization induced by in vitro ischemia. J Neurophysiol 90 : 3213-3223, 2003.

30. Kikuchi K, Kawahara K, Miyagi N, et al: Edaravone: a new therapeutic approach for the treatment of acute stroke. Med Hypotheses 75: 583-585, 2010.

31. Kikuchi K, Uchikado H, Miyagi N, et al: Beyond neurological disease: New targets for edaravone (Review). Int J Mol Med 28: 899-906, 2011

32. Kikuchi K, Takeshige N, Miura N, et al: Beyond free radical scavenging: Beneficial effects of edaravone (Radicut) in various diseases (Review). Exp Ther Med 3: 3-8, 2012

33. Kikuchi K, Kawahara K, Uchikado H, et al: Potential of edaravone for neuroprotection in neurologic diseases that do not involve cerebral infarction (Review). Exp Ther Med 2: 771-775, 2011

34. Kikuchi K, Miura N, Morimoto Y, et al: Beneficial effects of the free radical scavenger edaravone (Radicut) in neurologic diseases. J Neurol Neurophysiol 1: 1-5, 2011

35. Edaravone Acute Infarction Study Group: Effect of a novel free radical scavenger, edaravone (MCI-186), on acute brain infarction. Randomized, placebo-controlled, double-blind study at multicenters. Cerebrovasc Dis 15: 222-229, 2003.

36. Naritomi H, Moriwaki H, Metoki N, et al: Effects of edaravone on muscle atrophy and locomotor function in patients with ischemic stroke: a randomized controlled pilot study. Drugs RD 10: $155-163,2010$
37. Shinohara Y, Saito I, Kobayashi S and Uchiyama S: Edaravone (radical scavenger) versus sodium ozagrel (antiplatelet agent) in acute noncardioembolic ischemic stroke (EDO trial). Cerebrovasc Dis 27: 485-492, 2009.

38. Unno Y, Katayama M and Shimizu H: Does functional outcome in acute ischaemic stroke patients correlate with the amount of free-radical scavenger treatment? A retrospective study of edaravone therapy. Clin Drug Investig 30: 143-155, 2010.

39. Wahlgren N, Ahmed N, Davalos A, et al: Thrombolysis with alteplase 3-4.5 $\mathrm{h}$ after acute ischaemic stroke (SITS-ISTR): an observational study. Lancet 372: 1303-1309, 2008.

40. Hacke W, Kaste M, Bluhmki E, et al: Thrombolysis with alteplase 3 to 4.5 hours after acute ischemic stroke. N Eng J Med 359: $1317-1329,2008$

41. Wardlaw JM, Murray V, Berge E, et al: Recombinant tissue plasminogen activator for acute ischaemic stroke: an updated systematic review and meta-analysis. Lancet 379: 2364-2372, 2012.

42. Intracerebral hemorrhage after intravenous t-PA therapy for ischemic stroke. The NINDS t-PA Stroke Study Group. Stroke 28: 2109-2118, 1997

43. Yamashita T, Kamiya T, Deguchi K, et al: Dissociation and protection of the neurovascular unit after thrombolysis and reperfusion in ischemic rat brain. J Cereb Blood Flow Metab 29: 715-725, 2009

44. Lukic-Panin V, Deguchi K, Yamashita T, et al: Free radical scavenger edaravone administration protects against tissue plasminogen activator induced oxidative stress and blood brain barrier damage. Curr Neurovasc Res 7: 319-329, 2010.

45. Yagi K, Kitazato KT, Uno M, et al: Edaravone, a free radical scavenger, inhibits MMP-9-related brain hemorrhage in rats treated with tissue plasminogen activator. Stroke 40: 626-631, 2009.

46. Zhang W, Sato K, Hayashi T, et al: Extension of ischemic therapeutic time window by a free radical scavenger, Edaravone, reperfused with tPA in rat brain. Neurol Res 26: 342-348, 2004.

47. Kano T, Harada T and Katayama Y: Attenuation of extravasation of tissue plasminogen activator by the free radical scavenger, edaravone: evaluation in a rat thromboembolic stroke model. Neurol Res 27: 499-502, 2005.

48. Isahaya K, Yamada K, Yamatoku M, et al: Effects of edaravone, a free radical scavenger, on serum levels of inflammatory biomarkers in acute brain infarction. J Stroke Cerebrovasc Dis 21: 102-107, 2012.

49. Nakamura T, Kuroda Y, Yamashita S, et al: Edaravone attenuates brain edema and neurologic deficits in a rat model of acute intracerebral hemorrhage. Stroke 39: 463-469, 2008.

50. Kamiya T and Abe K: Future neuroprotective strategies in the post-thrombolysis era - neurovascular unit protection and vascular endothelial protection. Rinsho Shinkeigaku 51: 305-315, 2011 (In Japanese)

51. Wahlgren N, Ahmed N, Eriksson N, et al: Multivariable analysis of outcome predictors and adjustment of main outcome results to baseline data profile in randomized controlled trials: Safe Implementation of Thrombolysis in Stroke-Monitoring Study (SITS-MOST). Stroke 39: 3316-3322, 2008

52. Yamaguchi T, Mori E, Minematsu K, et al: Alteplase at $0.6 \mathrm{mg} / \mathrm{kg}$ for acute ischemic stroke within 3 hours of onset: Japan Alteplase Clinical Trial (J-ACT). Stroke 37: 1810-1815, 2006.

53. Mori E, Minematsu K, Nakagawara J, Yamaguchi T, Sasaki M and Hirano T: Effects of $0.6 \mathrm{mg} / \mathrm{kg}$ intravenous alteplase on vascular and clinical outcomes in middle cerebral artery occlusion: Japan Alteplase Clinical Trial II (J-ACT II). Stroke 41: 461-465, 2010.

54. Eesa M, Schumacher HC, Higashida RT and Meyers PM: Advances in revascularization for acute ischemic stroke treatment: an update. Expert Rev Neurother 11: 1125-1139, 2011.

55. Frendl A and Csiba L: Pharmacological and non-pharmacological recanalization strategies in acute ischemic stroke. Front Neurol 2: 32,2011

56. Tanswell P, Modi N, Combs D and Danays T: Pharmacokinetics and pharmacodynamics of tenecteplase in fibrinolytic therapy of acute myocardial infarction. Clin Pharmacokinet 41: 1229-1245, 2002.

57. Verstraete M: Third-generation thrombolytic drugs. Am J Med 109: 52-58, 2000

58. Parsons M, Spratt N, Bivard A, et al: A randomized trial of tenecteplase versus alteplase for acute ischemic stroke. N Eng J Med 366: 1099-1107, 2012.

59. Misra V, El Khoury R, Arora R, et al: Safety of high doses of urokinase and reteplase for acute ischemic stroke. AJNR Am J Neuroraidol 32: 998-1001, 2011. 
60. Liberatore GT, Samson A, Bladin C, Schleuning WD and Medcalf RL: Vampire bat salivary plasminogen activator (desmoteplase): a unique fibrinolytic enzyme that does not promote neurodegeneration. Stroke 34: 537-543, 2003.

61. Schleuning WD: Vampire bat plasminogen activator DSPA-alpha-1 (desmoteplase): a thrombolytic drug optimized by natural selection. Haemostasis 31: 118-122, 2001.

62. Hacke W, Albers G, Al-Rawi Y, et al: The Desmoteplase in Acute Ischemic Stroke Trial (DIAS): a phase II MRI-based 9-hour window acute stroke thrombolysis trial with intravenous desmoteplase. Stroke 36: 66-73, 2005.
63. Furlan AJ, Eyding D, Albers GW, et al: Dose Escalation of Desmoteplase for Acute Ischemic Stroke (DEDAS): evidence of safety and efficacy 3 to 9 hours after stroke onset. Stroke 37: 1227-1231, 2006

64. Hacke W, Furlan AJ, Al-Rawi Y, et al: Intravenous desmoteplase in patients with acute ischaemic stroke selected by MRI perfusion-diffusion weighted imaging or perfusion CT (DIAS-2): a prospective, randomised, double-blind, placebo-controlled study. Lancet Neurol 8: 141-150, 2009.

65. Paciaroni M, Medeiros E and Bogousslavsky J: Desmoteplase. Expert Opin Biol Ther 9: 773-778, 2009. 